\title{
Meander Dipole Antenna design for Passive UHF RFID Tags
}

\author{
TANG Fang-Mei ${ }^{1, a}$, LI Jian-Cheng ${ }^{2, b}$, and LI Cong ${ }^{3, \mathrm{c}}$ \\ ${ }^{1,2,3}$ College of Electronic Technology and Engineering \\ National University of Defense Technology, Changsha410003, China \\ a Email :15200833423@126.com, ${ }^{\mathrm{b}}$ Email:lijc_hh@126.com, ${ }^{\mathrm{c}}$ Email: cong.li@nudt.edu.cn
}

Keywords: dipole antenna, meander dipole, UHF RFID, T-match, passive tag

\begin{abstract}
A novel meander dipole antenna for passive UHF RFID tags, composed by normal meander dipole combined and ameliorated T-match network, is presented ,simulated and fabricated to be excellent performances, and suited accurately to complex impedance matching to the embedded microchip. Particular optimization is taken to the parameters of this dipole antenna by HFSS. This meander dipole is able to achieve a bandwidth of $120 \%$ for $\left|S_{11}\right|<-10 \mathrm{~dB}$ over the band of $500 \mathrm{MHz}-1.6 \mathrm{GHz}$, covering the entire frequency band of the global UHF RFID system(840MHz$960 \mathrm{MHz}$ ) dedicated for diverse regions. Especially in Chinese UHF RFID range of $900 \mathrm{MHz}$, maximum gain of $2.5 \mathrm{~dB}$, minimum return loss of $-56.5 \mathrm{~dB}$ and omnidirectional radiation are obtained, indicating prominent RF performances. The tested value basically agrees well with the simulated result.
\end{abstract}

\section{Introduction}

Radio Frequency Identification (RFID) in the ultrahigh frequency (UHF) bands is rapidly developing and has enormous potential application in Internet of Things (IOT) network. ${ }^{[1]}$ UHF RFID tag possesses particular capability to cope with complicated circumstances for its prominent advantages of expeditious identification over remote distance ${ }^{[2]}$ and tags anti-collision. ${ }^{[3]}$ Passive tags without power source indirectly derive power from RF signal; the micro-current in the antenna induced by the coming RF signal feeds adequate energy to the chip and the tag works. ${ }^{[4,5]}$ Actualization about the antenna matching to complex impedance chip nevertheless turns into a puzzling matter for tag antenna design. ${ }^{[3,6]}$

In RFID communication, on account of the directional sensitivity, the RF signal waves interfered with mediums, surroundings, locomotion, etc. can significantly affect the performances of tag antenna. ${ }^{[7]}$ Symmetrical dipole is one of typical forms to realize UHF, and structures of folded dipole, meander dipole and fractal dipole are developed. ${ }^{\left[{ }^{[3}\right.}$ These antennas can efficiently shrink the tag size, whereas the radiation characteristics damp in some degree. ${ }^{[1]}$ Fractal antenna therein has more serious recession in aerial radiation efficiency with the fractal parameters increasing.

The designed antenna is dedicated for a sort of UHF RFID tags independently contrived by our research group with the national standard (UHF bands of china: $840 \mathrm{MHz}-845 \mathrm{MHz}$ and $920 \mathrm{MHz}-925 \mathrm{MHz}$ ). Taking all the mentioned matters above into account, an antenna structure combining meander dipole with normal T-match network is given, simulated and fabricated in this paper. Aiming at making up shortfalls of traditional dipole antenna, the T-match network structure is revised in shape. The designed antenna achieves exact aerial matching to the given electronic tag chip UR3P2-T. Prominent RF performances in omnidirectional radiation, maximum E-field gain and return loss are illustrated in this paper. Besides, well tested result is obtained to be in accord with the simulated result.

\section{Antenna design and analysis}

To design an antenna of excellent performance, ensuring the chip impedance of low resistance and high capacitance to be exactly matched with antenna's to receive maximum energy; widening the bandwidth sufficient to contain the chip's working frequency; lessening blind area; minifying 
antenna size is where the shoe pinches. The matched tag chip UR3P2-T has a complex impedance of $5.5-\mathrm{j} 86 \Omega$ and can be activated at frequency range from $920.5 \mathrm{MHz}$ to $925 \mathrm{MHz}$.

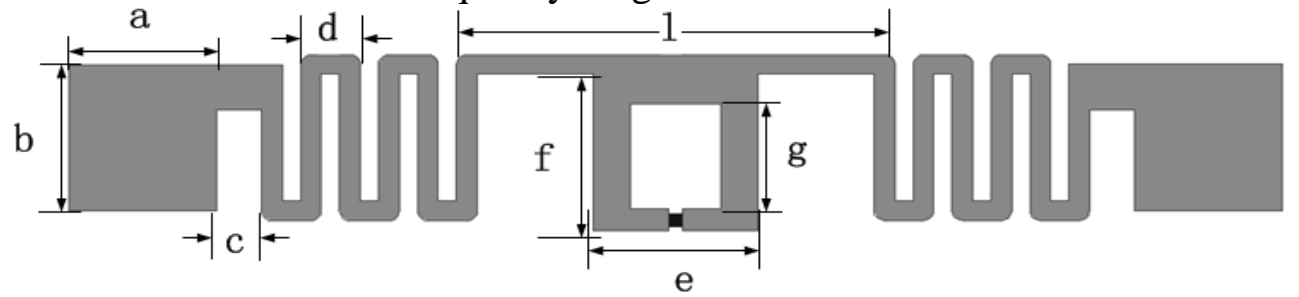

Fig.1 Meander dipole antenna designed by traditional methods

An antenna structure of meander dipole combined with T-match network is presented in Fig.1. Meander dipole plays as the main part of radiation structure, determining the antenna radiation characteristics including direction, resonant frequency and antenna gain. The T-match network is useful to adjust the antenna impedance exactly matched with the given chip.

Meander dipole antenna is radiated by two symmetrical meander dipole arms with multiple bend lines. The current on dipole is unequally distributed. The line units of diverse bend lines bring about wave-way difference, triggering phase shift to component the phase difference caused by current. Then electrical length of antenna descends for the same the resonant frequency. It is an effective way to obtain high imaginary antenna impedance and compact structure as well.

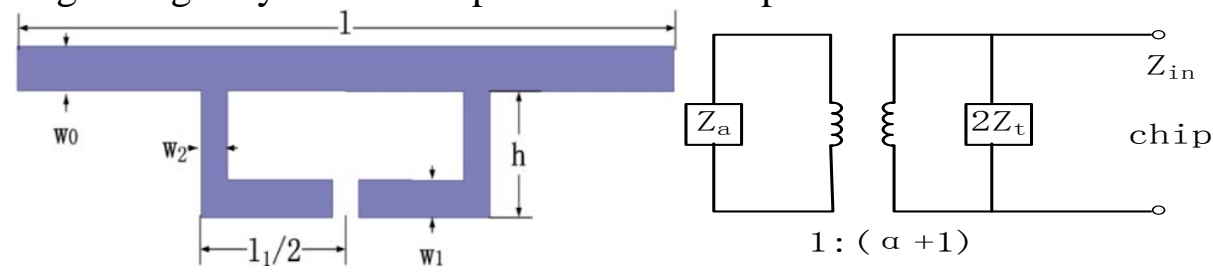

Fig.2 planar T-match network and the corresponding equivalent circuit

Normal T-match network in Fig.2 can be considered as connection of long dipole and a center-fed short dipole by transmitting lines at the shorting terminals. ${ }^{[7]}$ The shorting line should be less than $\lambda / 4$ to achieve inductive impedance at the feed port. The input impedance of terminal transmitting line can be given by ${ }^{[9]}$

$$
\begin{aligned}
& Z_{t}=j Z_{0} \tan \left(\pi \frac{w_{2}+l_{1}}{\lambda}\right) \\
& Z_{0} \approx 276 \log \frac{4 h}{\sqrt{w_{0} w_{1}}}
\end{aligned}
$$

The current branches into two paths by proportion of $\alpha$ at shorting point, the input impedance of long dipole is coupled with T-match network in proportion of $1:(1+\alpha)$. The resulting input impedance of the whole antenna $\mathrm{Z}_{\text {in }}$ seen from the feed port is

$$
Z_{\text {in }}=\frac{2 Z_{t}(1+\alpha)^{2} Z_{a}}{2 Z_{t}+\left(1+\alpha^{2}\right) Z_{a}}
$$

Where, $\mathrm{Z}_{\mathrm{a}}$ is the dipole center impedance without any matching network. This structure plays an equivalent role as an impedance converter, adjusting the antenna impedance to the certain rang so as to realize conjugated matching between antenna and chip. Thus, diminutive antenna return loss is acquired.

The antenna must also provide a good matching to chip for maximum power transfers. A definition about the power reflection coefficient is given by: ${ }^{[10]}$

$$
\mathrm{S}_{11}=\frac{Z_{\text {in }}-Z_{c}^{*}}{Z_{\text {in }}+Z_{c}}
$$

Where $Z_{c}$ is the impedance of connected electronic chip. In order to match this chip, the matching antenna impedance should be designed at $5.5+\mathrm{j} 86 \Omega$ to counteract the capacitive part of chip.

\section{Result}

The designed antenna is simulated by Ansys HFSS, and the results of the antenna performance are illustrated. 


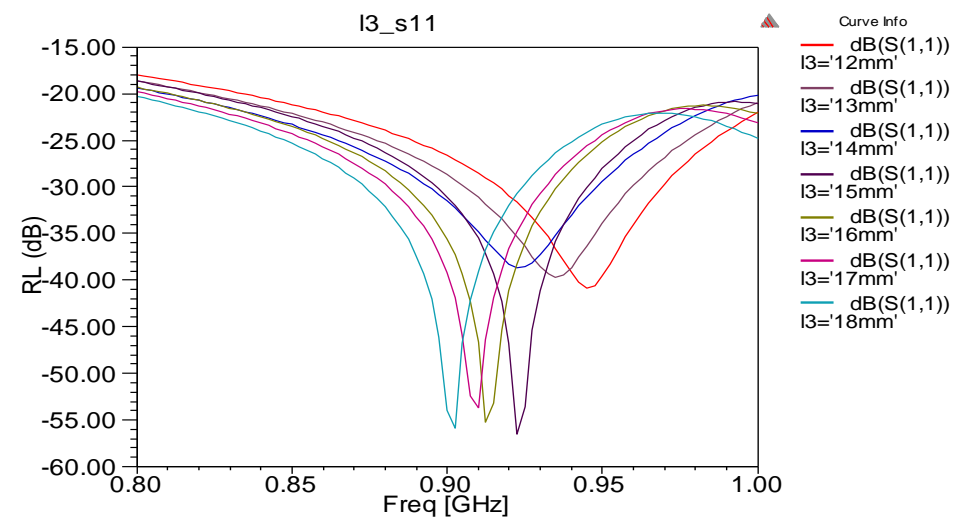

Fig. 3 the impact of 13 to the resonant frequency $(13=1 / 2)$

The resonant frequency falls with 13 increasing, shown in Fig. 3. It is reachable to make the antenna resonant around the designing frequency by proper selection of the electrical length of dipole. It can be observed through Fig. 3 that the antenna resonant at the goal frequency of $922.5 \mathrm{MHz}$ when $l 3$ is valued $15 \mathrm{~mm}(l=30 \mathrm{~mm})$.

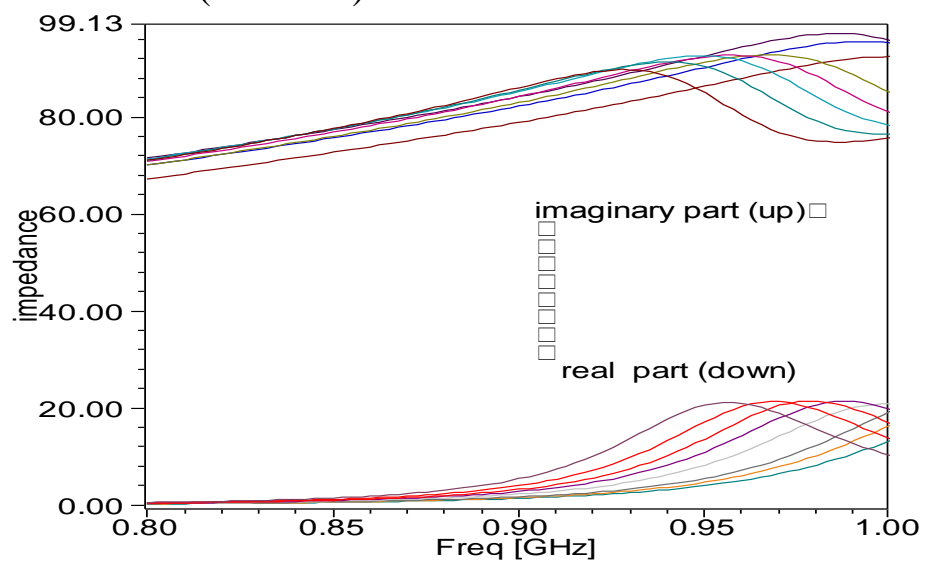

Fig. 4 the impact of $b$ to impedance

The meander lines of the dipole can help realize high imaginary antenna impedance. The impact of the meander line height $b$ is illustrated in Fig. 4 that high Q value (high imaginary part and low real part) is achieved in the meander dipole structure.

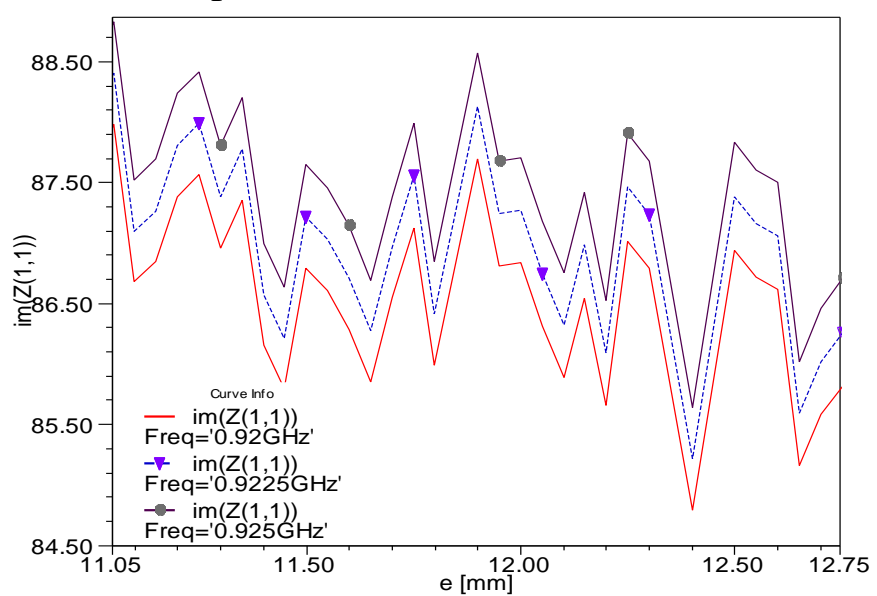

Fig. 5 Adjustment for exact matching impedance by parameter $e$

The parameter $e$ is used for length adjustment of the short dipole in the T-match network, shown as Fig.5. The width of the short dipole meliorates the proportion of coupling impedance, under guarantee the permitting excursion about resonant frequency of $922.5 \mathrm{MHz}$.

Tune the electrical length $(a, c, d, l)$, meandering height $b$ and the bending number of the dipole to make the antenna resonant at the center frequency of $922.5 \mathrm{MHz}$; consulting formulas (1) $(3)$, make optimization to the parameters of the T-match structure for an utmost approaching to the design impedance goal. The final parameters of the structure dimension are listed in Lab.1 
Table. 1 the parameters in antenna design (unit: $\mathrm{mm}$ )

\begin{tabular}{c|c|c|c|c|c|c|c}
\hline $\mathrm{a}$ & $\mathrm{b}$ & $\mathrm{c}$ & $\mathrm{d}$ & $\mathrm{e}$ & $\mathrm{f}$ & $\mathrm{g}$ & $\mathrm{l}$ \\
\hline 10 & 11 & 3.3 & 2.9 & 12.2 & 11.2 & 7.7 & 30 \\
\hline
\end{tabular}

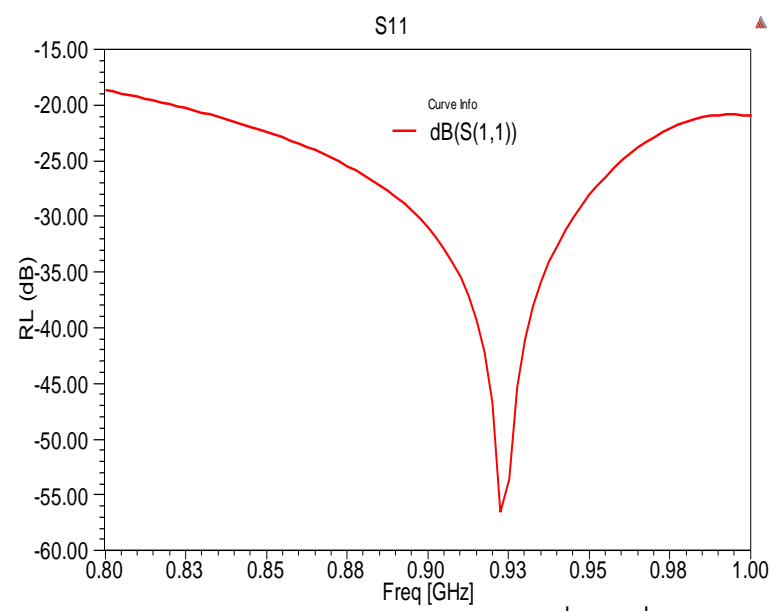

(a) Return loss trail $\left|S_{11}\right|$

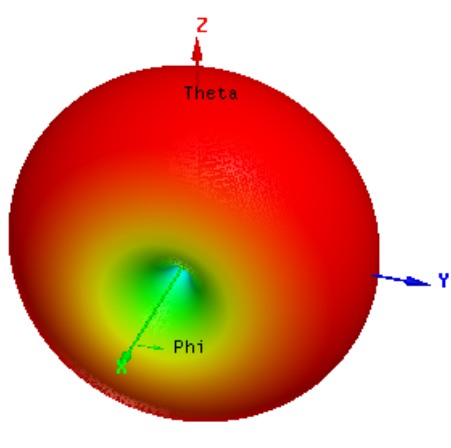

(c) 3D \&2D antenna Gain pattern

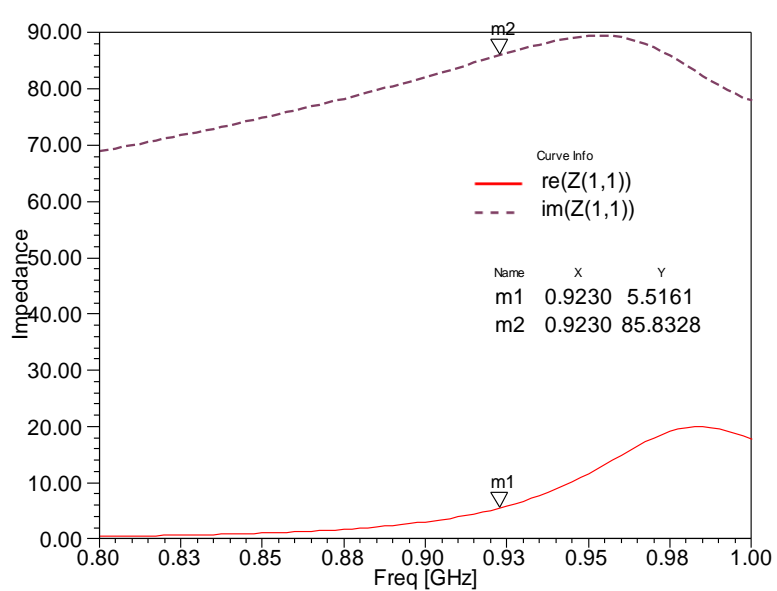

(b) The impedance of the antenna

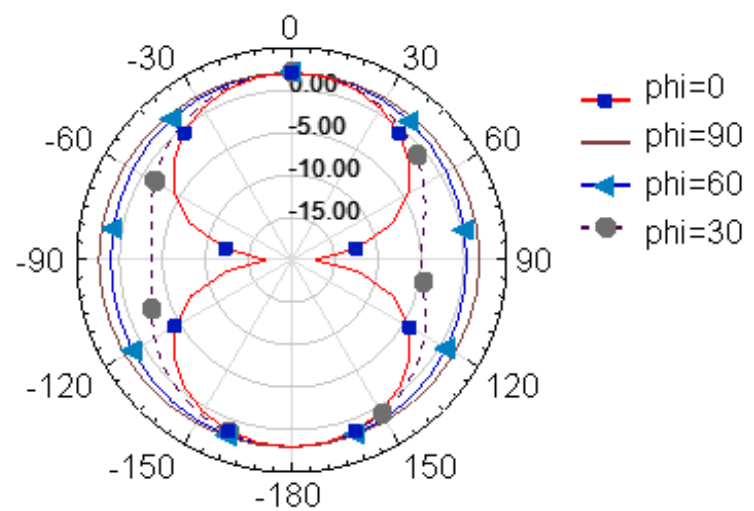

Fig.6 Aerial performances of the meander dipole antenna:

In Fig.6, the return loss trail $\left|S_{11}\right|$ in (a) illustrates that the antenna is resonant around $922.5 \mathrm{MHz}$ and the corresponding return loss is $-56.5 \mathrm{~dB}$. The designed antenna impedance of $(5.5+j 85.8) \Omega$ is shown in (b), achieves an exceeding conjugate matching with the given electronic tag chip at its working frequency. 3D and 2D gain patterns of the antenna are shown in (c), indicating the favorable omnidirectional radiation and $\mathrm{E}$ field maximum gain of $2.5 \mathrm{~dB}$. The designed structure is also fabricated using copper foil on a piece of paper, as shown in Fig.7. The measured return loss in Fig.8, achieves agreement with the simulated result in principle. The discrepancy is mainly caused by the limits of fabricating precision, the effect of paper permittivity and measuring dithering by the vector network analyzer. 
(b)

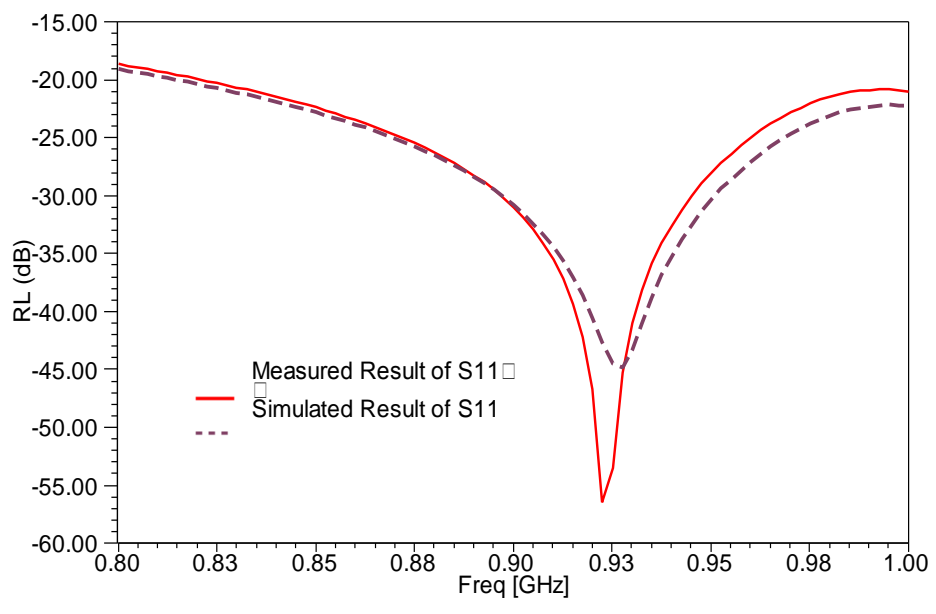

Fig. 7 (a) Photograph of the fabricated RFID tag antenna and (b) Compare of $S_{11}$ between measured and simulated results

\section{Conclusion}

A novel meander dipole antenna for passive UHF RFID tags is proposed. Basing on the traditional designing way, the amelioration has been done by increasing the transmitting shape in the T-match network for low return loss and high radiation gain. The wideband obtained by this antenna has proved to cover the entire frequency band dedicated for the global UHF RFID system. Especially in Chinese band of $900 \mathrm{MHz}$, the antenna characteristic of $\left|S_{11}\right|$ can descended to $-56.5 \mathrm{~dB}$. The impact of several key parameters has been analyzed to help make optimization. The factual antenna was fabricated on a piece of paper and tested to be satisfactory. The designed structure has exactly conjugated matched with the connected chip UR3P2-T of impedance (5.5-j86) $\Omega$ and showed prominent aerial performances. Further research effort could not be spared to improve the workmanship for small and cheap antennas in quantity, such as printing antennas with aluminum on paper, better dedicated for the RFID application.

\section{Acknowledgment}

The author wishes to appreciate for the support of the National Natural Science Foundation of China (grant No. 61201167)

\section{References}

[1] Simone G and Agostino M: IEEE Anten. and Wireless Propag. Let. Vol. 9(2011), p. 1225

[2] Kerem K and Catherine D: IEEE International Conference on Electronics, Circuits and Systems, 2013, p. 823

[3] Zamora G, Zuffanelli S, Paredes F, Martin F and BonacheJ: IEEE Transactions on Microwave Theory and Techniques Vol. 61(2013), p. 4090

[4] Ahmed A S and Kayder M Q: 7th International Multi-Conference on Systems, Signals and Devices, 2010, p. 1

[5] Etienne P, Smail T and Rail S: Proceedings of the IEEE Vol. 100(2012), p. 2330

[6]Da Costa f c, De Lima E R, Yoshioka R T, Bertuzzo J E and Koeppe J: Microwave and Optoelectronics Conference, 2013, p. 1

[7] Marrocco G: Anten. and Propag. Magazine Vol. 50(2008), p. 66

[8] Chen H M, Chen H D, Chen C H and Chen J S: Anten. and Propag. Society International Symposium, 2013, p. 1740 
[9] Reich M T and Bauer R C: IEEE International conference on RFID, 2014, p. 23

[10] Kerem K and Catherine D: IEEE RFID Technology and Applications Conference, 2014, p. 591 